\title{
Significance of Schistosomal Granuloma Modulation
}

\section{Luciana M Silva, André LM Fernandes, Aryon Barbosa Jr, Irismar R Oliveira, Zilton A Andrade ${ }^{+}$}

Centro de Pesquisas Gonçalo Moniz-Fiocruz, Rua Valdemar Falcão 121, 40295-001 Salvador, BA, Brasil

Hepatic Schistosoma mansoni periovular granulomas undergo changes in size, cellular composition and appearance with time. This phenomenom, known as "immunological modulation", has been thought to reflect host immunological status. However, as modulation has not been observed outside the liver, participation of local factors, hitherto little considered, seems crucial. Components of the extracellular matrix of periovular granulomas of the mouse were particularly studied in three different organs (liver, lung and intestine) and during three periods of infection time (acute, intermediate and chronic) by means of histological, biochemical and imunofluorescence techniques, while quantitative data were evaluated by computerized morphometry, in order to investigate participation of local factors in granuloma modulation. Results confirmed modulation as a exclusively hepatic phenomenom, since pulmonary and intestinal granulomas, formed around mature eggs, did not change size and appearance with time. The matricial components which were investigated (Type I, III and IV collagens, fibronectin, laminin, proteoglycans and elastin) were found in all granulomas and in all organs examined. However, their presence was much more prominent in the liver. Elastin was only found in hepatic granulomas of chronic infection. The large amount of extracellular matrix components found in hepatic granulomas was the main change responsible for the morphological aspects of modulation. Therefore, the peculiar environment of the liver ultimately determines the changes identified in schistosomal granuloma as "modulation".

Key words: modulation - granuloma - shistosomiasis - extracellular matrix

Hepatic granulomas formed around mature eggs of Schistosoma mansoni undergo changes in size, appearance and cellular constitution with time. This has been interpreted as a mechanism of host protection, with the host reactions becoming more economical and efficient during chronic infection (Andrade \& Warren 1964). The immunological counterpart has been thoroughly investigated, both in vivo (Colley 1981, Chensue et al. 1993, Bogen et al. 1995) and in vitro (Doughty \& Phillips 1982, Parra et al. 1991), including its cytokine patterns (Wynn \& Cheever 1995, McKerrow 1997). However, there are data indicating that granulomas outside the liver may behave differently. Granulomas in the intestine were observed to be already modulated in the ileum from the very beginning, although modulation was said to occur in the colon and ileal Peyer's patch (Weinstock \& Boros 1981, 1983). In the lungs granulomas presented

Supported by Papes 2 (Fiocruz) and Pronex.

${ }^{+}$Corresponding author. Fax: +55.71.356.4292. E-mail: zilton@cpqgm.fiocruz.br

Received 21 May 1999

Accepted 13 January 2000 the same morphology regardless the time of infection (Vidal et al. 1992, Eltoum et al. 1995). Such findings suggest that the so-called "immunological modulation" of periovular granulomas in schistosomiasis is mainly influenced by local factors. If this is so, the current interpretation of the immunological modulation concept needs to be revised.

To further investigate this possibility, a comparative study of size, appearance and matrix composition of granulomas formed in three different organs of mice (liver, intestine and lung), during three different periods of infection (early, intermediate and late), was undertaken. Methods of histopathology, immunofluorescence, biochemistry and morphometry were applied in an attempt to understand the real significance of the so-called "immunological modulation" of schistosomal granuloma.

\section{MATERIALS AND METHODS}

Thrity-one albino Swiss mice of both sexes, weighing 18-23 g, maintained with free access to a commercial balanced diet and water, were used. Animals were submitted to transcutaneous infection with 50 recently eliminated $S$. mansoni cercariae each. Later, animals were randomly divided into three groups, according to time of infection: first group (acute phase), animals sacrificed eight 
weeks following cercarial exposure; second group (intermediate phase), animals sacrificed at the 16th week after exposition; and third group (chronic phase), animals sacrificed at the 22nd weeks of infection.

To augment the chances of finding pulmonary granulomas, an extra group of mice was infected with 100 cercariae. Five animals were included in the acute phase group, and another five in the intermediate group. No animals infected with 100 cercariae survived to be included in the chronic group.

At the time of sacrifice the animals were anesthetized with ether, the abdomen was opened and the aorta severed. The liver was removed and weighed. The counting of worms was performed by examining the mesenteric veins and by smashing the liver between two slides. In this last case, several fragments to be submitted to other techniques were previously saved. A lobe of the lung was fixed by intra-tracheal injection of $10 \%$ formalin. Fragments of the liver, small intestine and lung were used for the following purposes:

Histology - Tissues were fixed in either $\mathrm{pH} 7.2$ phosphate buffered $10 \%$ formalin in PBS or in Bouin's fluid. The $5 \mu$-thick paraffin sections obtained were stained with the following methods: hematoxylin and eosin, sirius-red for collagen, Gomori' s silver impregnation for reticulum and Alcian-blue staining under two different $\mathrm{pHs}$ (2 and 7).

Counting of eggs - Fragments of the liver and intestines were weighted and digested overnight in $4 \%$ potassium hydroxide and the eggs counted according to Cheever's method (1970). Results were expressed as concentration of eggs per gram of tissue.

Immunofluorescence - Fragments of liver, intestines and lung were placed in small carton boxes filled with Tissue-Tek (Miles, USA) and immediately frozen in liquid nitrogen for 5 min. Blocks were then kept in air-thight plastic bottles at $-80^{\circ} \mathrm{C}$ until sectioned in a criostat at $-20^{\circ} \mathrm{C}$. The sections were collected on clean glass slides and immediately washed in iced saline and treated with either one of the following anti-sera: anti-collagen I, anticollagen III, anti-collagen IV, anti-fibronectin, antilaminin, and anti-desmin. All anti-sera were kindly supplied by Dr Jean-Alexis Grimaud. The anti-sera were derived from human material and were prepared in rabbits. Their dilution at the moment of use varied from 1:50 and 1:100. Details about their preparation and specificity can be found elsewhere (Andrade \& Grimaud 1986). The secondary antibody was a fluoresceinated anti-rabbit IgG (Sigma), diluted at 1:40 in a weak solution of Evans blue. Controls consisted of sections treated with saline only and sections treated with an irrelevant serum (normal rat serum).

Hydroxyproline measurements - Fragments of the liver were weighed and then submitted to biochemical determination of hydroxyproline content by the colorimetric method B of Bergman and Loxley (1963). Samples weighing from 100 to 250 $\mathrm{mg}$ were used. Hydroxyproline levels were corrected for intensity of infection by dividing total hepatic hydroxyproline (without correction for hydroxyproline levels in uninfected mice) by the number of $S$. mansoni eggs in the liver.

Morphometry - Histological sections either stained with hematoxylin and eosin or sirius-red were analyzed by automatic morphometry using a Leica Q500MC Image Processing and Analysis System (Leica Cambridge, Cambridge, England). For morphometric measurements a total sectional area of $17.01 \times 10^{6} \mu \mathrm{m}^{2}$ per case was evaluated (Coutinho et al. 1997).

Statistical analysis - Numerical values obtained from egg counting, hydroxyproline measurement and morophometric data were analyzed using parametric tests. Analysis of variance with one or two factors (Anova) was used for identification of significance. Student-Newman-Keuls and the Student$\mathrm{t}$ tests were applied for group differences. Results revealing $\mathrm{p}<0.05$ were considered significant.

\section{RESULTS}

An average of five worm pairs per mouse was recovered, and that decreased to three during the intermediate and chronic phases of infection. When 100 cercariae were used, twice as many worms were counted. The number of eggs per gram of tissue was greater in the liver $(14,713)$ than in the intestines $(8,451)$, this difference being statistically significant $(\mathrm{p}<0,05)$. However, when morphometric measurements for numerical density were considered, the values for the intestine were greater than those for the liver (Table I). Morphometry also showed that the volume of the granulomas, considered as being spherical, decreased significantly in the liver, especially when those from the acute phase were compared with the chronic phase. No significant differences in granuloma volume were noted for those formed in the intestine or lung (Table II). Regarding volume density, which means the total volume occupied by the granulomas within a given unit of volume, the values for the liver were greater during the acute phase, decreasing thereafter. Such decrease did not occur for the intestine or lung. During the acute phase, granuloma volume density for the liver was greater than for the intestine and lung, but during the intermediate and chronic phases such difference lacked statistical significance (Table III). 
TABLE I

Values for numerical density ( $\mathrm{Nn}$ ) of granulomas in different organs of mice infected with 50 Schistosoma mansoni cercariae during three periods of time, seven animals in each group

\begin{tabular}{lccc}
\hline Organs & \multicolumn{3}{c}{ Time of } \\
\cline { 2 - 4 } & 8 weeks & 16 weeks & 22 weeks \\
\hline Lungs & $1,36 \pm 1,78$ & $3,29 \pm 4,35$ & $5,38 \pm 5,77$ \\
Liver & $12,24 \pm 2,09$ & $14,89 \pm 2,89$ & $18,53 \pm 4,29^{a}$ \\
Intestines & $15,59 \pm 2,96$ & $16,48 \pm 2,42$ & $21,93 \pm 7,21^{b}$ \\
\hline
\end{tabular}

$a$ : $\mathrm{P}<0,05$ (Student-Newman Keuls) as compared to 8 weeks; $b$ : $\mathrm{p}<0,05$ (Student-Newman Keuls) as compared to 8 and 16 weeks.

TABLE II

Average values for granuloma volume in different organs and at different periods of time in mice infected with 50 Schistosoma mansoni cercariae, seven animals in each group

\begin{tabular}{|c|c|c|c|}
\hline \multirow[t]{2}{*}{ Organs } & \multicolumn{3}{|c|}{ Time of infection $\left(\times 10^{4} \mu \mathrm{m}^{3}\right)$} \\
\hline & 8 weeks & 16 weeks & 22 weeks \\
\hline Lungs & $17,62 \pm 27,62$ & $44,28 \pm 43,23$ & $32,56 \pm 39,19$ \\
\hline Liver & $481,53 \pm 161,51$ & $252,98 \pm 227,66$ & $84,32 \pm 26,22^{a}$ \\
\hline Intestines & $22,96 \pm 15,13$ & $46,71 \pm 17,13$ & $16,96 \pm 4,49$ \\
\hline
\end{tabular}

$a$ : $\mathrm{p}<0,05$ (Student-Newman Keuls) as compared to 8 and 16 weeks.

\section{TABLE III}

Values for volume density $(\mathrm{Vv})$ of periovular granulomas in different organs and at different periods of time in mice infected with 50 Schistosoma mansoni cercariae, seven animals in each group

\begin{tabular}{lccc}
\hline Organs & \multicolumn{3}{c}{ Time of infection } \\
\cline { 2 - 4 } & 8 weeks & 16 weeks & 22 weeks \\
\hline Lungs & $0,00 \pm 0,00$ & $0,01 \pm 0,01$ & $0,01 \pm 0,02$ \\
Liver & $0,18 \pm 0,05$ & $0,012 \pm 0,05$ & $0,07 \pm 0,02^{a}$ \\
Intestines & $0,04 \pm 0,02$ & $0,09 \pm 0,03 \mathrm{a}$ & $0,04 \pm 0,03$ \\
\hline
\end{tabular}

$a: \mathrm{p}<0.05$ (Student-Newman Keuls).

Histological evaluation was concerned only with periovular granulomas having a well preserved central miracidium. Old, involuting granulomas were not considered. Granulomas were abundant in sections from the liver. During the acute stage they appeared as predominantely exudative, frequently exhibiting a central halo of necrosis and a sprinkling of polymorphonuclear eosinophils (Fig. 1). Collagen fibers were thin and loosely arranged in a concentric disposition. Under immunofluorescence microscopy, Type III collagen appeared more abundant than type I, this latter being represented by fibers that seemed thicker and straight, rather than wavy, when compared with Type III collagen (Fig. 7A, B). Fibronectin formed a prominent network of fine fibrils all over the granuloma area (Fig. 7C). Type IV collagen and laminin were absent from periovular granulomas, revealing only basement mem- branes in small blood vessels situated at the granuloma periphery (Fig. 7D). A pale, diffuse Alcian blue staining marked all granulomas. This staining was similarly observed with either acidic and neutral $\mathrm{pH}$. Within the structures of the miracidia, the Alcian blue staining became more accentuated, especially when a neutral $\mathrm{pH}$ was employed.

During the intermediate phase hepatic granulomas differed from those of the acute phase in showing a tendency to confluence and in being smaller and with the cells and collagen fibers becoming more packed. Eosinophils were less numerous while macrophages and fibroblasts became more prominent.

At the chronic stage periovular granulomas were especially characterized by being smaller, well delimited and more fibrotic than before (Fig. 2). Collagen fibers were thicker, concentrically distributed, and strongly fluorescent when tested 


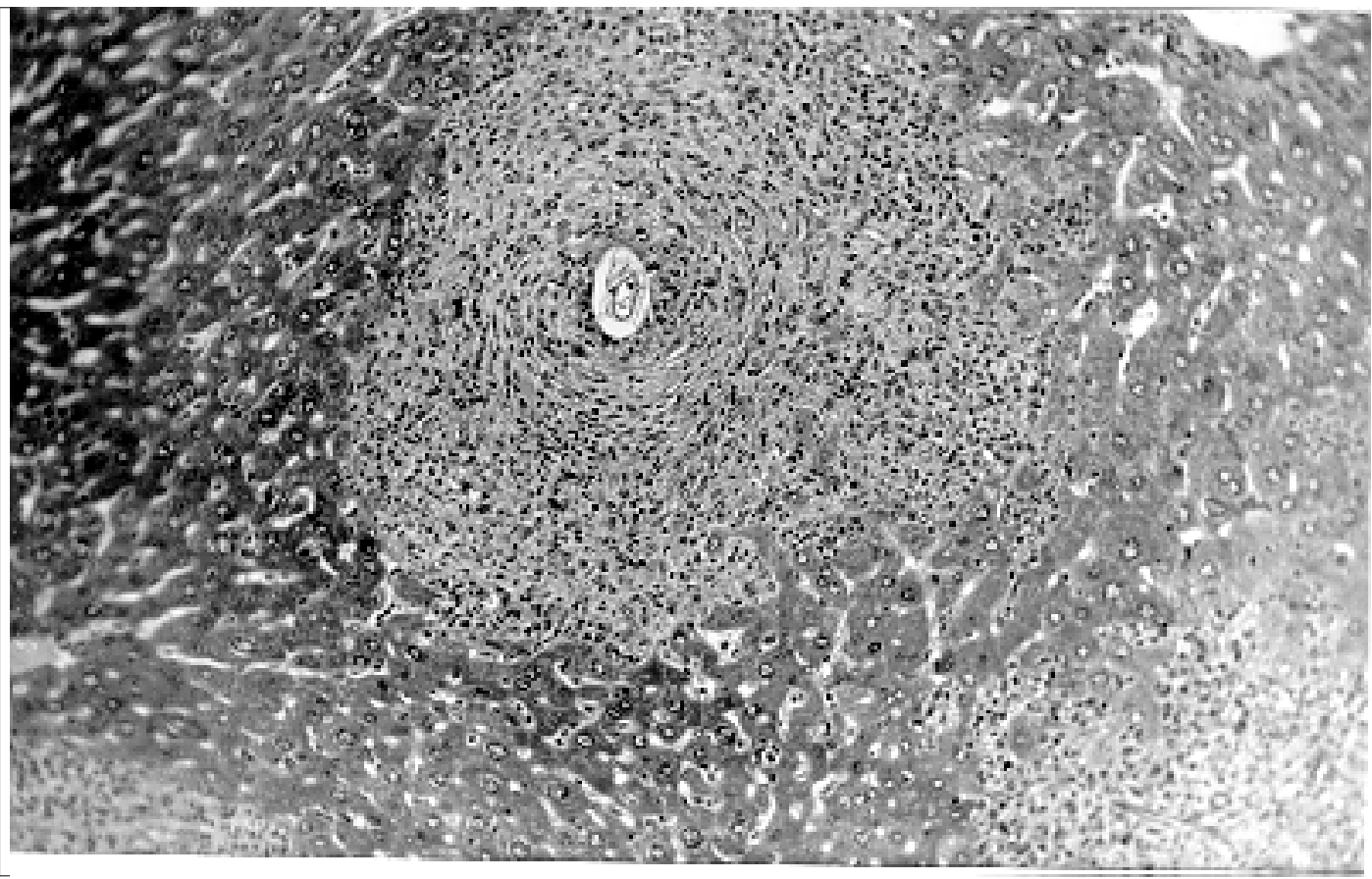

Fig 1: acute phase periovular granuloma in the liver. A large and irregular inflammatory area appears around a central schistosome egg. Central necrosis is present, although scanty. H \& E, 64X

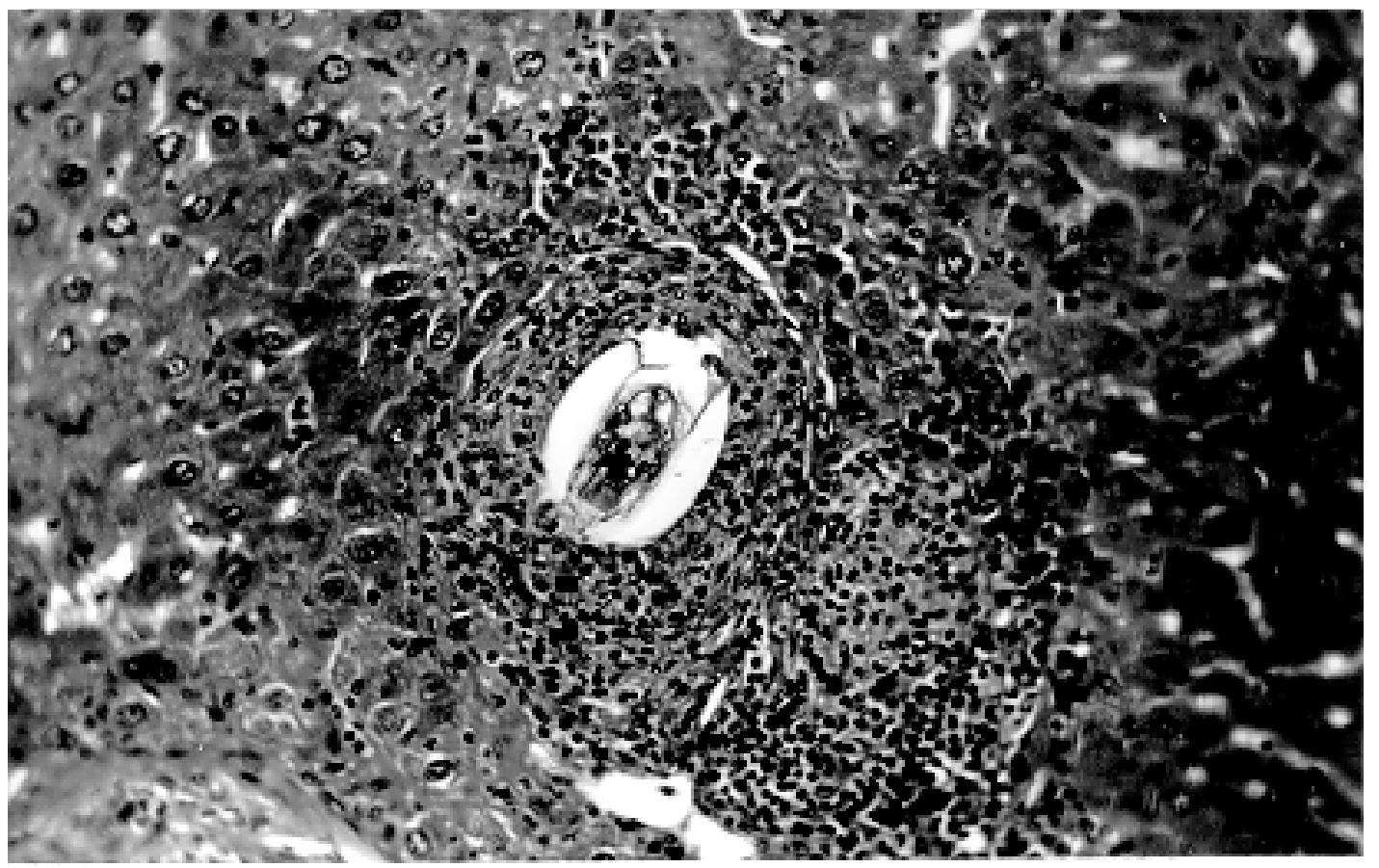

Fig 2: chronic phase periovular granuloma. Reaction around the egg is composed of macrophages and fibroblasts, with a few eosinophils. Central necrosis is absent. H \& E, 200X. 
for the presence of type I and type III collagens. The staining for fibronectin was less intense than that obtained for the acute phase. Orcein-positive elastic fibers, conspicously absent in acute and intermediate phase granulomas, appeared in a few granulomas of the chronic phase (Fig. 3). Desminpositive cells increased their numbers proportionally from acute to chronic phase.

Granulomas formed around mature eggs in the intestine and lung showed a mixture of proliferative and exudative features, but they tended to maintain the same morphologic aspect when examined at different times of infection (Figs 4, 5). All components of extracellular matrix revealed by immunofluorescence microscopy in the liver were also present in granulomas of intestine and lung, although always in smaller amount (Fig. 7E, F).

Hydroxyproline content was evaluated only for the liver. Data expressed as $\mu \mathrm{mol}$ of hydroxyproline per gram of tissue versus number of eggs per gram of liver are depicted in Fig. 6A, B, C. Positive correlation occurred at the acute phase only. However, the concentration of hydroxyproline in the liver increased progressively with time of infection. The average numbers were: normal liver $3.42 \mu \mathrm{mol}$; acute phase $6.90 \mu \mathrm{mol}$, intermediate phase $7.84 \mu \mathrm{mol}$ and chronic phase $8.77 \mu \mathrm{mol}$.

\section{DISCUSSION}

Present findings confirm and extend previous data which are suggestive that the so-called "immunological modulation" of schistosomal periovular granuloma is a morphological phenomenom peculiar to the liver (Grimaud et al. 1987, Vidal et al. 1992). Granulomas formed around eggs injected directly into the lungs (Lichtenberg 1962) or liver (Edungbola \& Schiller 1979) differ accordingly with the previous immunological state of the host, that is, whether the recipient animal is already $S$. mansoni infected or not. However, injections of eggs into normal animals or even into those already infected (Domingo \& Warren 1968), create an artificial and complex situation, with some peculiar features that differ from those of a cercarial infection, as has been discussed by Cheever et al. (1998).

Weinstock and Boros $(1981,1983)$ observed differences in size and cellular composition of granulomas formed at different intestinal segments. Probably these differences were small. Present data refer to the intestines as a whole, and no evidences of modulation were noted in that organ during different periods of infection.

Quantitative data about granuloma size and volume were subjected to morphometric evalua-

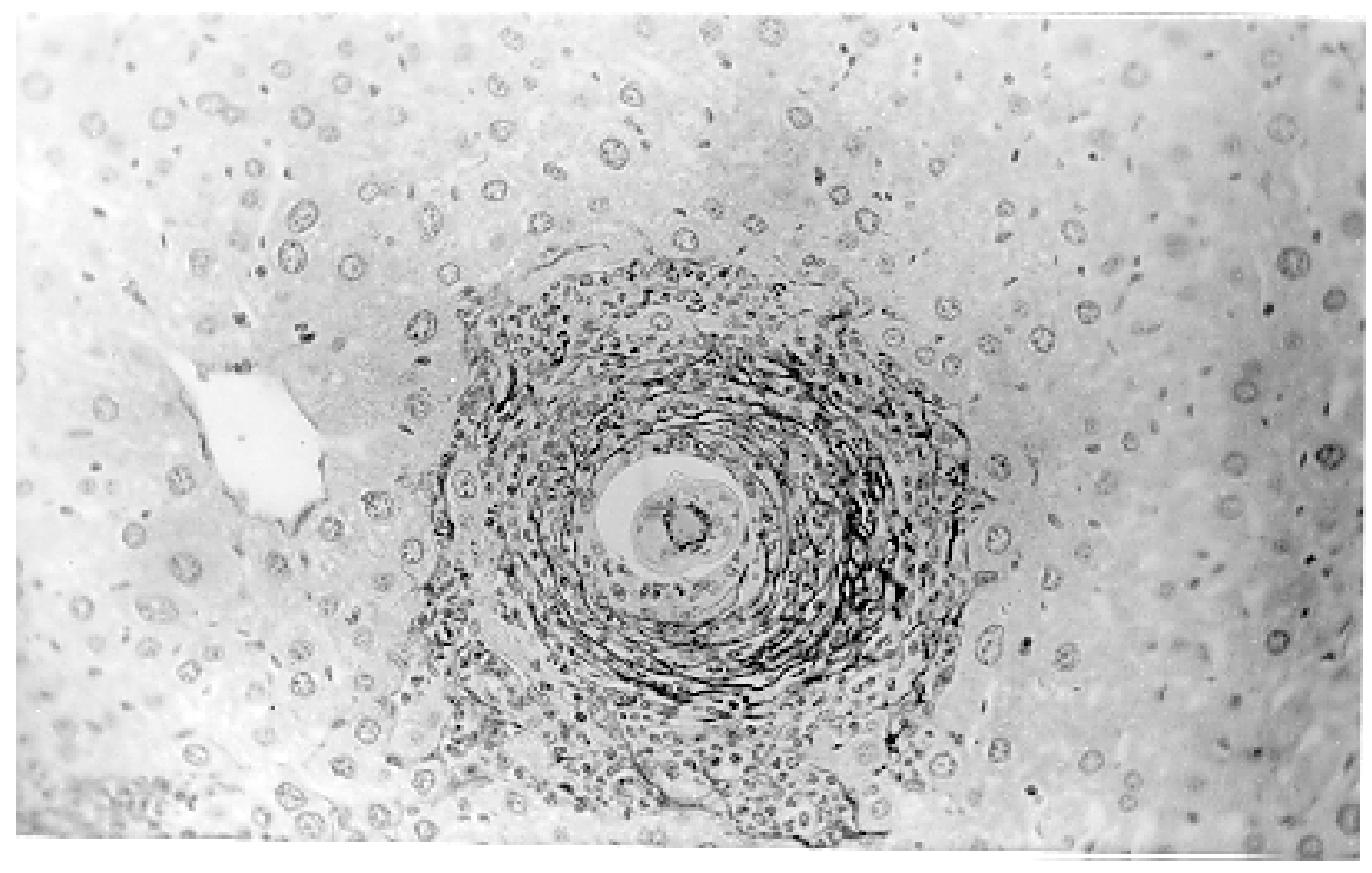

Fig. 3: a well delimited inflammatory reaction around a schistosome egg formed during the chronic phase of infection, exhibits fine, concentric, black, orcein-positive elastic fibers. Orcein method for elastic fibers, 200X. 


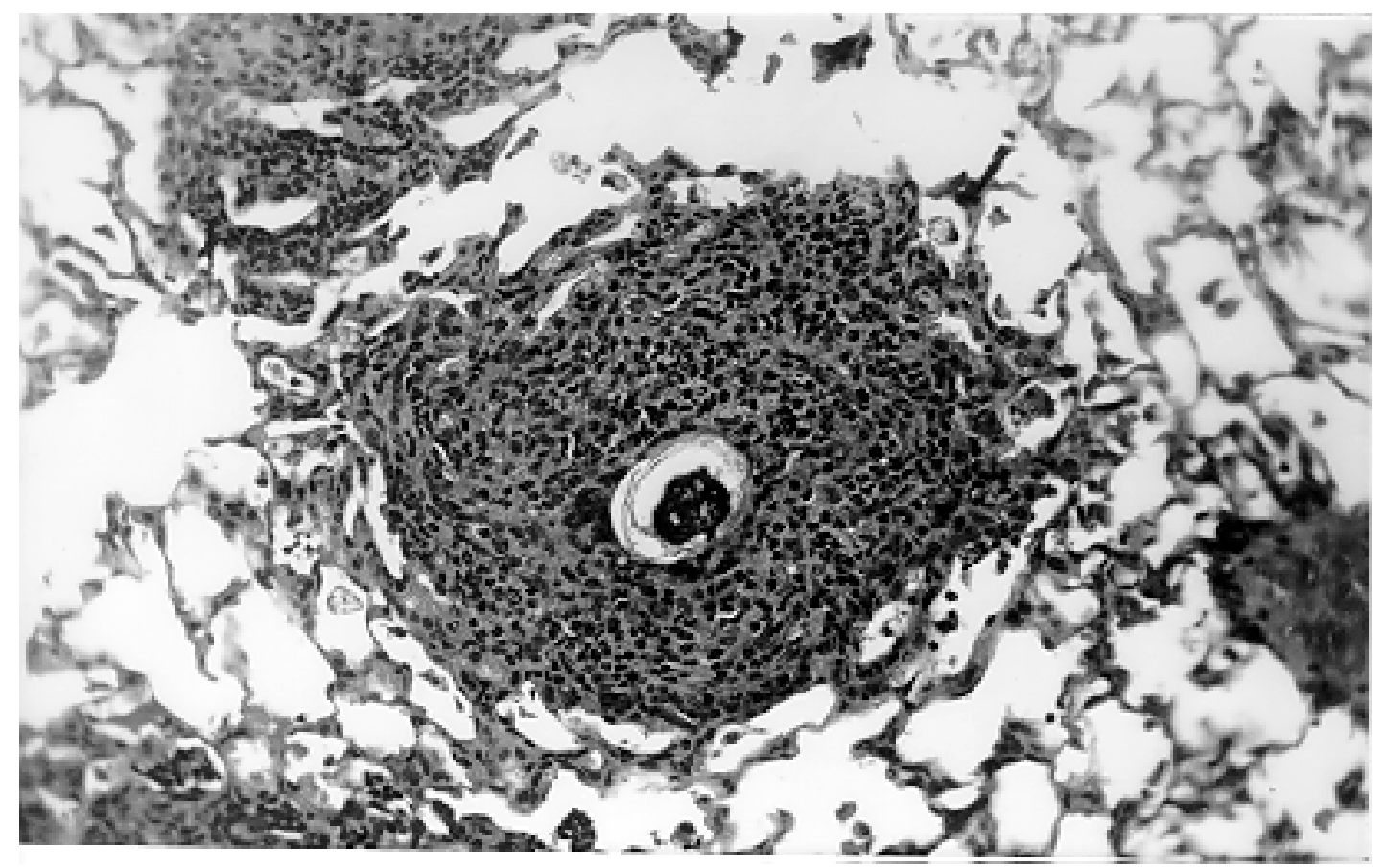

Fig 4: a mixture of exudative and proliferative features characterizes periovular granulomas formed within the pulmonary alveolar tissue. Intermediate phase of infection. H \& E, 200X.

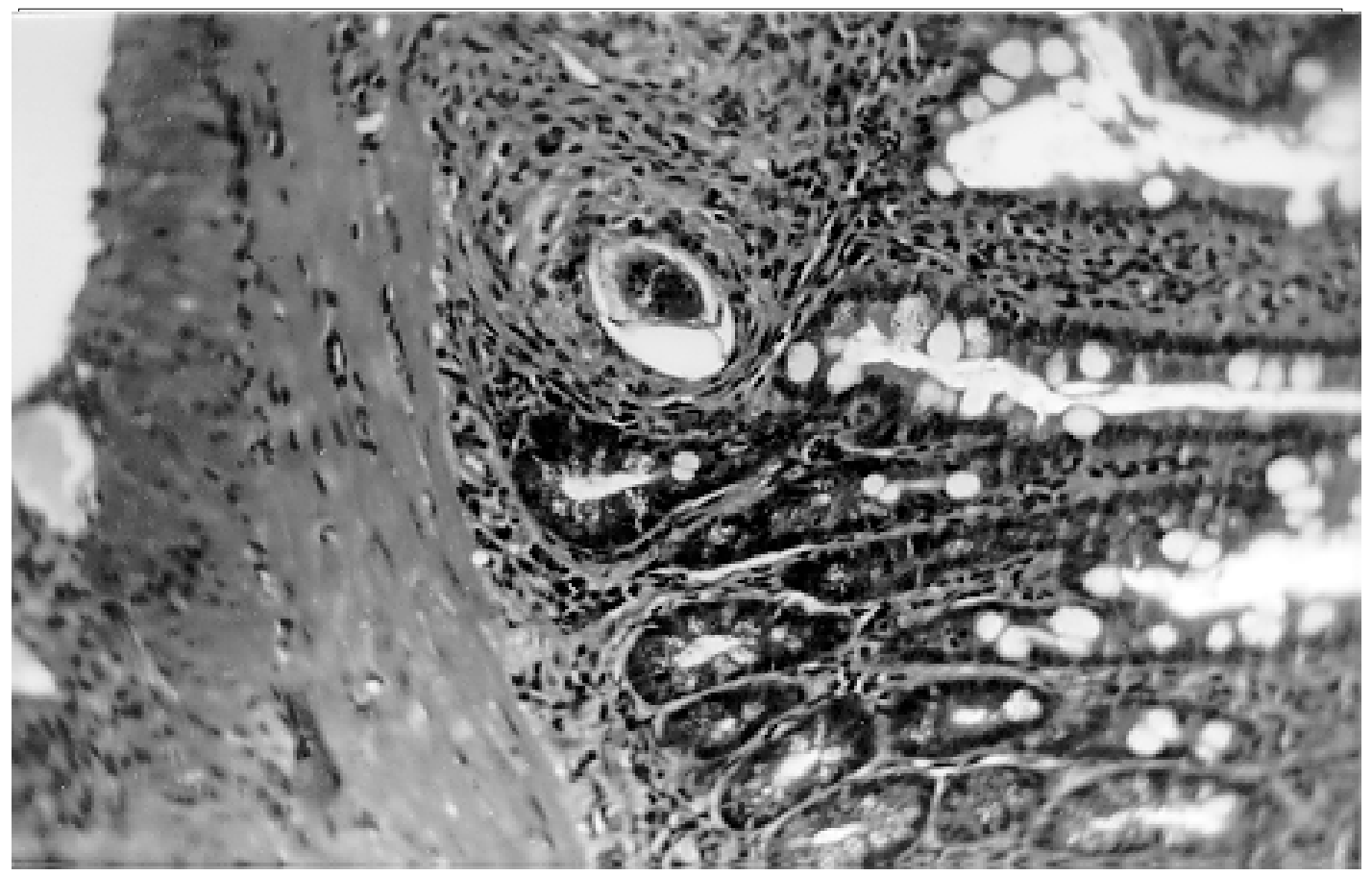

Fig 5: a small granuloma formed around a mature Schistosoma mansoni egg in the intestinal submucosa, during the acute period of infection. $\mathrm{H} \& \mathrm{E}, 200 \mathrm{X}$. 
A

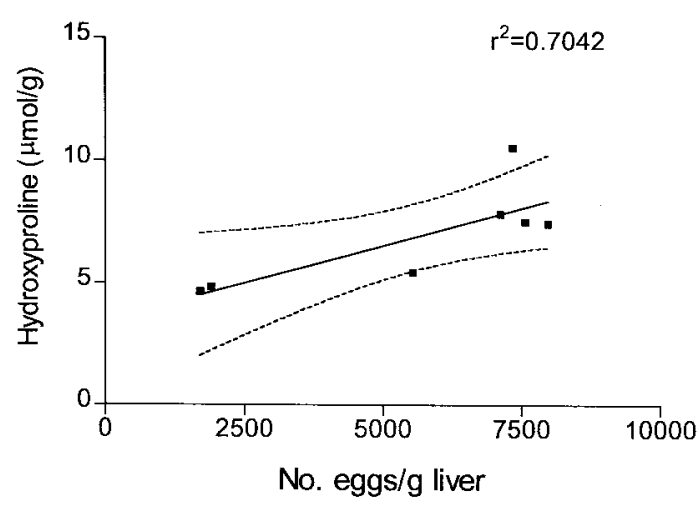

B

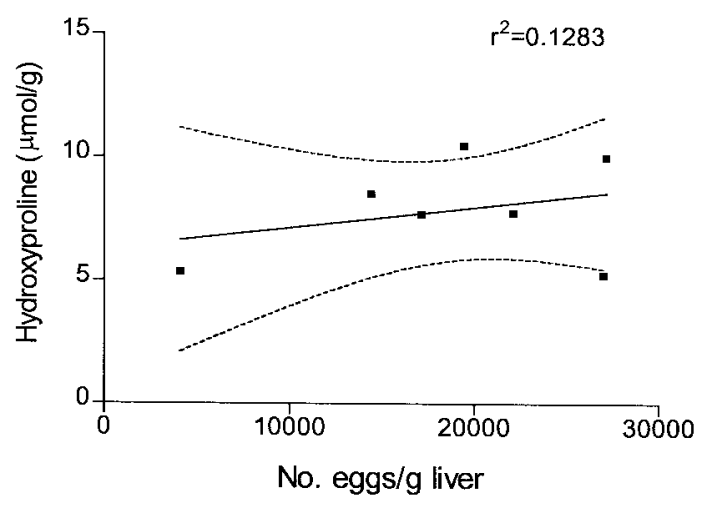

C

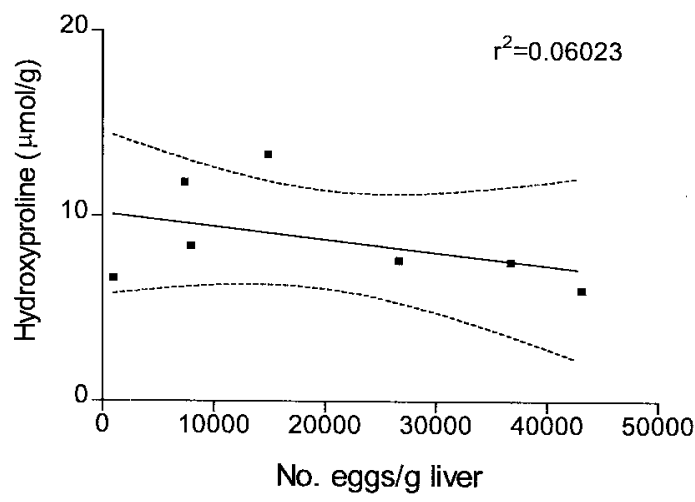

Fig 6: correlation between the number of eggs and hydroxyproline content in the liver of mice infected with 50 Schistosoma mansoni cercariae at different periods of infection. A: acute phase ( 8 weeks); B: intermediate phase (16 weeks); C: chronic phase (22 weeks). tion, and the nature of granulomatous extracellular matrix was analyzed by immunofluorescent microscopy. Results indicated that changes, or lack of it, regarding size, appearance and cellular composition of granulomas located at different organs and at different times would mostly depend on local factors. The influence of general factors, such as immune cells, cytokine patterns and so on, is certainly important. However, more attention should be paid to the peculiar cellular population of the liver and the extracellular matrix it can produce and degrade. The matricial components which were investigated (Type I, III and IV collagens, fibronectin, laminin and proteoglycans) were also found in all granulomas and in all organs examined. However, their presence was much more prominent in the liver. As a matter of fact, elastic fibers (elastin) were found only in hepatic granulomas, and exclusively during the chronic stage of infection, but that finding was the only one which was qualitatively different from the granulomas formed elsewhere. Although elastic fibers were abundant in portal space fibrosis due to schistosomiasis in man, periovular granulomas were said to be devoid of such fibers (Junqueira et al. 1986, Andrade \& Freitas 1991). Anyway, quantitatively, the large amount of extracellular matrix found in hepatic granulomas was the main change responsible for the morphologic aspects of modulation. Probably, the presence of special cellular elements, such as, hepatocytes, Ito cells and Kupffer cells, which are known to coordinately play an important role in formation and degradation of extracellular matrix in the liver (Blomhoff \& Wake 1991, Casu et al. 1994, Gressner \& Bachem 1994, Enzan et al. 1995) accounts for the peculiar way periovular granulomas are modulated in that organ.

\section{REFERENCES}

Andrade ZA, Freitas LAR 1991. Hyperplasia of elastic tissue in hepatic schistosomal fibrosis. Mem Inst Oswaldo Cruz 86: 447-456.

Andrade ZA, Grimaud JA 1986. Evolution of the schistosomal hepatic lesions in mice after curative chemotherapy. Am J Pathol 124: 59-65.

Andrade ZA, Warren KS 1964. Mild prolonged schistosomiasis in mice: alterations in host response with time and the development of portal fibrosis. Trans $R$ Soc Trop Med Hyg 58: 53-57.

Bergman I, Loxley R 1963. Two improved and simplified methods for the spectrophotometric determination of hydroxyproline. Anal Chem 35: 1961-1965.

Blomhoff R, Wake K 1991. Perisinusoidal stellate cells of the liver: important roles in retinol metabolism and fibrosis. FASEB 5: 271-277.

Bogen EA, Flores Villanueva PO, Mccusker ME, Fogelman I, Garifallou M, El-Attar ER, Kwan P, Stadecker MJ 1995. In situ analysis of cytokine re- 

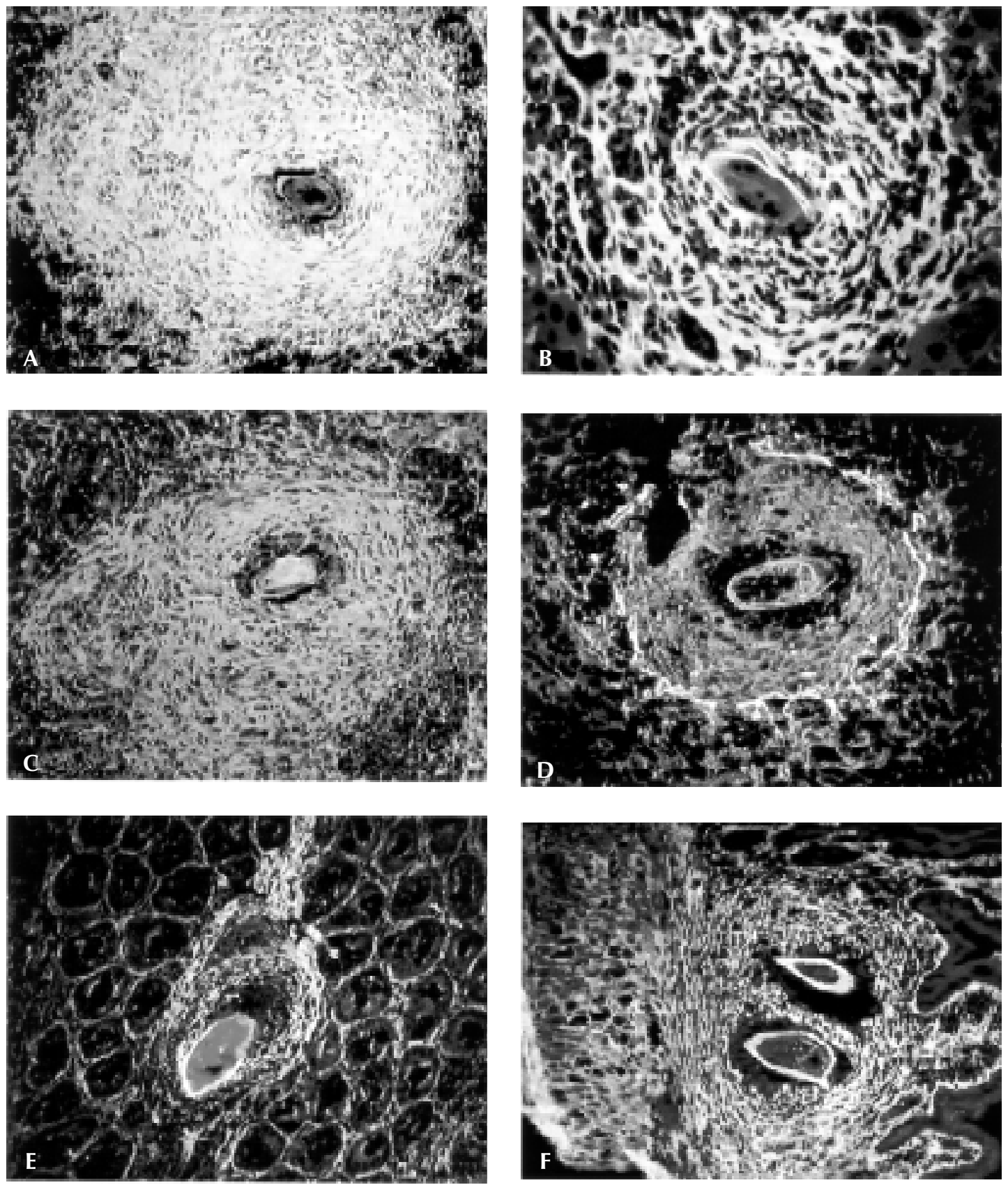

Fig 7: components of the extracellular matrix are depicted in the periovular granulomas by immunofluorescence. A: liver, type I collagen, acute phase of the infection, 200X; B: liver, type III collagen, intermediate phase, 400X; C: liver, fibronectine, acute phase, 200X; D: liver, laminine, acute phase, 200X. Note presence of blood vessel at the granuloma periphery (arrows) E: intestine, type I collagen, intermediate phase, 200X; F: intestine, fibronectine, chronic phase, 200X.

sponses in experimental murine schistosomiasis. $L a b$ Invest 73: 252-258.

Casu A, Canepa M, Nanni G 1994. Le cellule stellate perisinusoidali o cellule di Ito ed il loro roulo nella fibrosi epatica. Pathologica 86: 467-499.

Cheever AW 1970. Relative resistance of the eggs of human schistosomes to digestion in potassium hydroxide. Bull WHO 43: 601-603.

Cheever AW, Jankovic D, Yap GS, Kullberg MC, Sher A, Wynn TA 1998. Role of cytokines in the formation and downregulation of hepatic circumoval granulomas and hepatic fibrosis in Schistosoma 
mansoni-infected mice. Mem Inst Oswaldo Cruz 93 Suppl. I: 25-32.

Chensue SW, Warmington KS, Hershey SD, Terebuh PD, Othman M, Kunkel SL 1993. Evolving T-cell responses in murine schistosomiasis. Th2 cells mediate secondary granulomatous hypersensitivity and are regulated by $\mathrm{CD} 8+\mathrm{T}$ cell in vivo. J Immunol 151: 1391-1400.

Colley DG 1981. T lymphocytes that contribute to the immunoregulation of granuloma formation in chronic murine schistosomiasis. J Immunol 126: 1465-1468.

Coutinho EM, Souza MM, Silva LM, Cavalcanti REA, Barbosa Júnior A, Cheever AW, Andrade ZA 1997. Pathogenesis of schistosomal "pipestem" fibrosis: a low-protein diet inhibits the development of "pipestem" fibrosis in mice. Int J Exp Pathol 78: 337-342.

Domingo EO, Warren KS 1968. Endogenous desensitization: changing host granulomatous response to schistosome eggs at different stages of infection with Schistosoma mansoni. Am J Pathol 52: 369-379.

Doughty BL, Phillips SM 1982. Delayed hypersensitivity granuloma formation around Schistosoma mansoni eggs in vitro. I. Definition of the model. $J$ Immunol 128: 30-36.

Edungbola LD, Schiller LE 1979. Histopathology of hepatic and pulmonary granulomata experimentally induced with eggs of Schistosoma mansoni. J Parasitol 65: 253-261.

Eltoum IA, Wynn TA, Poindexter RW, Finkelman FD, Lewis FA, Sher A, Cheever AW 1995. Suppressive effect of IL-4 neutralization differs for granulomas around Schistosoma mansoni eggs injected into mice compared to eggs laid in infected mice. Infect Immun 69: 2532-2536.

Enzan H, Himeno H, Iwamura T, Saibara T, Onishi S, Yamamoto Y, Miyazaki E, Hara H 1995. Sequential changes in human Ito cells and their relation to postnecrotic liver fibrosis in massive and submassive hepatic necrosis. Wirchows Arch 426: 95-101.

Gressner AM, Bachem MG 1994. Cellular communications and cell-matrix interactions in the pathogen- esis of fibroproliferative diseases: liver fibrosis as a paradigm. Ann Biol Clin 52: 205-226.

Grimaud JA, Boros DL, Takiya C, Mathew RC, Emonard $\mathrm{H}$ 1987. Collagen isotypes, lamin, and fibronectin in granulomas of liver and intestines of Schistosoma mansoni-infected mice. Am J Trop Med Hyg 37: 335344.

Junqueira LCU, Montes GE, Toledo OMS, Joazeiro PP 1986. Morphological, histochemical and biochemical observations on the connective tissue matrix of in situ and isolated hepatic granulomas in experimental murine schistosomiasis. Ann Trop Med Parasitol 80: 27-41.

Lichtenberg F Von 1962. Host response to eggs of $S$. mansoni I. Granuloma formation in the unsensitized laboratory mouse. Am J Pathol 41: 711-713.

McKerrow JH 1997. Cytokine induction and exploitation in schistosome infections. Parasitology 115: 107-112.

Parra JC, Gazzinelli G, Goes AM, Moyes RB, Rocha R, Colley DG, Doughty BL 1991. Granulomatous hypersensitivity to Schistosoma mansoni egg antigens in human schistosomiasis. II. In vitro granuloma modulation induced by polyclonal idiotypic antibodies. J Immunol 147: 3949-3954.

Vidal MRFS, Barbosa Júnior AA, Andrade ZA 1992. Exprimental pulmonary schistosomiasis: lack of morfological evidence of modulation in schistosomal pulmonary granulomas. Rev Inst Med Trop $S$ Paulo 33: 423-429.

Weinstock JV, Boros DL 1981. Heterogeneity of the granulomatous response in the liver, colon, ileum, and ileal Peyer's patches to schistosome eggs in murine schistosomiasis mansoni. J Immunol 127: 1906-1909.

Weisntock JV, Boros DL 1983. Organ-dependent differences in composition and function observed in hepatic and intestinal granulomas isolated from mice with schistosomiasis mansoni. J Immunol 130: 418422.

Wynn TA, Cheever AW 1995. Cytokine regulation of granuloma formation in schistosomiasis. Curr Opin Immunol 7: 505-510. 